\title{
Treatment of Degenerative Disc Disease associated with Modic changes, with ozone therapy. A new therapeutic proposal.
}

\section{Anibal Martin Grangeat, Angeles Erario}

IAOT (Instituto Argentino de Ozonoterapia)

\section{OPEN ACCESS}

\section{Citation}

Martin Grangeat A, Erario A, Treatment of Degenerative Disc Disease associated with Modic changes, with ozone therapy. A new therapeutic proposal. J Ozone Ther. 2020:4(5)

doi: 10.7203/jo3t.4.5.2020.14739

\section{Academic Editor \\ Jose Baeza-Noci, \\ School of Medicine, Valencia \\ University, SPAIN \\ Editor \\ World Federation of Ozone Therapy, \\ Brescia, ITALY}

\section{Received}

April 20, 2019

\section{Accepted}

July 20, 2019

\section{Published \\ December 15, 2020}

\section{Intellectual Property}

Martin Grangeat A.

This is an open access article distributed under the terms of the Creative Commons Attribution License (CC BY 4.0), which permits unrestricted use, distribution, and reproduction in any medium, provided the original author and source are credited.

\section{Authors information}

anibalgrangeat@iaot.com.ar

\section{ABSTRACT}

Modic Changes (MCs) represent signal changes in the vertebral endplate and subchondral bone region visualized on T1 and T2 weighted magnetic resonance images (MRI). Three types of MCs It have been identified (Types 1 to 3). Modic changes are commonly associated with low-back pain (LBP).

In the last few years scientific papers have shown that one of the causes of MCs (fundamentally, the MCs type 1) is the infection caused by an anaerobic bacterium of very low virulence: Propionibacterium acnes (P. acnes). This bacterium resides on the human skin, oral cavity, intestinal tract, and external ear canal as normal flora. As a consequence of tooth brushing and endodontic-therapy, could invade the blood stream leading to transient bacteremia. The authors propose a treatment which consists of Amoxicillin-clavulanate $(500 \mathrm{mg} / 125 \mathrm{mg})$ three times a day, at 8-hour intervals, for 90 days/100 days.

\section{Objectives}

Ozone therapy could be the gold standard therapy for MC1 because ozone is a powerful anti-microbial, anti-inflammatory and analgesic agent, which regulates oxidative stress, immune system and oxygen metabolism.

\section{Materials and Methods}

Patients with LBP lasting 6 months or more and MC1 confirmed by MRI of lumbar spine L3/L4 or L4/L5 or L5/S1 and who responded with 5 or more points on the VAS scale were treated with three intradiscal sessions of ozone therapy and ten paravertebral sessions.

\section{Results}

Patients who were treated with ozone therapy according to our treatment proposal showed a substantial improvement on VAS comparing the beginning and the end of the treatment. They show a significant difference in pain, and general condition. However, these results were not reflected on ODI scale, neither on the MRI but we could notice certain improvements.

Key words: Modic changes, degenerative disc disease, ozone therapy, low back pain.

\section{Introduction}

Modic changes $(\mathrm{MC})$ are a common phenomenon on magnetic resonance imaging (MRI) in spinal degenerative diseases and strongly linked with low back pain (LBP). Histology, radiology, potential mechanisms, natural history and clinical studies of MC has formed the foundation on which our understanding of spinal degenerative diseases is built.

Signal intensity changes of vertebral endplates and subchondral bone are 
often observed in magnetic resonance imaging (MRI) in the patients with spinal degenerative diseases. In 1988, Modic et al. summarized these changes and classified them into three types, and then modic changes (MC), as a medical term, were used in the studies on spinal degenerative diseases. (1)

$\mathrm{MC}$ are bone marrow and endplate lesions visible on MRI. Type I changes demonstrate decreased signal intensity on T1-weighted images and increased signal intensity on T2-weighted images. Histopathologic sections of disks with type I changes show disruption and fissuring of the endplate and vascularized fibrous tissues within the adjacent marrow, prolonging T1 and T2. Type II changes are represented by increased signal intensity on T1-weighted images and isointense or slightly hyperintense signal on T2-weighted images. Disks with type II changes also show evidence of endplate disruption, with lipid marrow replacement in the adjacent vertebral body. Type III changes are represented by a decreased signal intensity on both T1-and T2-weighted images and correlate with extensive bone sclerosis on plain radiographs. The lack of signal in the type III change reflects the relative absence of marrow in areas of advanced sclerosis (2-3).

Modic changes (bone edema) in vertebrae are observed in $6 \%$ of the general population and in 35-40 \% of people with low back pain. These changes are strongly associated with low back pain. A recent systematic review showed that the prevalence for any type of $\mathrm{MC}$ in patients with chronic non-specific low back pain (CLBP) was $46 \%$ as opposed to $6 \%$ in the general population. (4)

In 2001 something "changes" because Stirling et al hypothesized that the inflammation seen around the nerve root in patients with sciatica could be caused by microbial infection. In this work published in The Lancet they used a serological test to diagnose deep-seated infections caused by low virulent grampositive microorganisms. 43 of $140(31 \%)$ patients with sciatica tested positive. Intervertebral disc material from a further 36 patients with severe sciatica who had undergone microdiscectomy was cultured for the presence of microorganisms. 19 of these patients $(53 \%)$ had positive cultures after long-term incubation. Propionibacterium acnes was isolated from 16 of the 19 (84\%) positive samples. Low virulent microorganisms, in particular $\mathrm{P}$. acnes, might be causing a chronic low-grade infection in the lower intervertebral discs of patients with severe sciatica. P. acnes is an anaerobic, gram-positive and rod-shaped bacterium. Resides on the human skin, oral cavity, intestinal tract, and external ear canal as normal flora (5) these microorganisms could have gained access to the spinal disc after previous minor trauma. In this preliminary study they have detected raised concentrations of a specific serum antibody to a glycerophospholipid (lipid S), an exocellular bacterial cell wall component. So, they postulated that patients with sciatica have a breach in the mechanical integrity of a spinal disc, possibly from minor trauma, which allows access by low virulent microorganisms, stimulating a chronic inflammatory response with accompanying symptoms. They thought that previous epidural injections could be a further source of microorganisms. However, 53\% from whom bacteria were isolated had not had a pre-operative epidural injection, and $41 \%$ of the tissue culture negative patients had. These findings suggest that epidural injections were not the source of the microorganisms. The results also suggest that $P$. acnes, might be associated with chronic low-grade infection in the lower intervertebral discs of patients with severe sciatica. The long generation time of Propionibacterium makes these microorganisms ideal candidates for chronic infection. $P$ acnes produces many exocellular virulence factors (including lipase, proteinase, hyaluronidase, neuramidase, and phospholipase $\mathrm{C}$ ), which may contribute to its pathogenicity. These microorganisms may cause prosthetic hip infections, but also might be associated with the inflammation seen in sciatica, and may possibly even be a primary cause of this disorder. (6) 
But is necessary point that since the initial description of Modic type 1 vertebral endplate changes there has been ongoing debate regarding their cause. Different studies have attributed Modic type 1 vertebral endplate changes to traumatic injury to the vertebral endplate (7), localized action of proinflammatory mediators $(\mathbf{7}, \mathbf{8})$, and low-grade bacterial infection (6-9). So, Modic changes type 1 are secondary to injury, inflammation, or infection? (10).

The possible theories on the pathogenesis of Modic Type 1 vertebral endplate changes are well hypothesized in a Crockett's et all. (10) work. They described the three possible mechanism and how they are connected between them. The mechanical stress model shows that increased shear forces acting on the vertebral endplate lead to endplate microtrauma, with the resultant marrow edema correlating with the Modic type 1 vertebral endplate changes seen on MRI. In the inflammatory model elevated levels of proinflammatory mediators, such as IL-6, TNFa, and prostaglandin E2 in disk tissue are described. Crockett proposed the internal disk disruption hypothesis which is, that repeated trauma to intervertebral disks causes release of inflammatory mediators within the nucleus pulposus that then diffuse through the vertebral endplates into the adjacent marrow instigating the inflammatory process (8). And finally, the infection model, in which it was suggested that disk disruption allows passage of low-virulence anaerobic bacteria into the disk. These bacteria thrive in the anaerobic environment, resulting in a lowgrade bacterial discitis with the Modic type 1 vertebral endplate changes visible on MRI representing the associated reactive marrow edema. Furthermore, one study showed the presence of low-virulence anaerobic bacteria in a far higher proportion of patients with Modic changes type 1 than in those with disk herniation alone (4), and another study showed that treatment of patients with Modic changes type 1 and low back pain with a long course of antibiotics was significantly more effective than placebo in all outcome measures (9).

However, subsequent systematic reviews found that, although evidence was limited, the link between disk infection and degeneration is possible and there was moderate evidence suggesting that bacteria were both associated with and a causative agent of Modic type 1 changes $(10,11,12)$. Crocket propose that the three processes are in fact closely linked. Several processes may instigate a chain of events leading to the MRI findings of Modic changes type 1. Mechanical disruption of the vertebral disk and endplate due to minor subclinical trauma appears to be the initial unifying step in the process. This results in an inflammatory response characterized by edema within the endplates and adjacent marrow that may be amplified by exposure of the marrow to substances originating from the intervertebral disk. Proinflammatory material from the disk nucleus may diffuse into the adjacent marrow leading to a cascade of inflammatory mediators, further edema, and neovascularization. In addition, the disrupted endplate and increased vascular permeability in the inflamed tissue may allow diffusion of low-virulence bacteria, present in the bloodstream of certain patients, into the disk. The anaerobic environment within the disk is an ideal environment for these bacteria and further propagates the peridiscal marrow inflammation. These processes are linked and are not mutually exclusive (10).

In a case-control study of animal model of Modic changes (MCs) on rabbits, ZhiShan et all, evaluated the feasibility of inducing of MCs by injection of Propionibacterium acne (P. acnes) into the lumbar intervertebral discs of rabbits. Their results showed that in-vivo P. acnes infection of the disc can induce degeneration of the disc and an inflammatory response in the endplate region, presenting as MCs type I and II time dependently (13). They observed bone mineral density loss and trabecular bone quality reduction in the endplate regions by micro-CT, which is consistent with the bone resorption, probably the result of catabolic activation induced by P. acnes. 
The bone resorption could be the result of both osteoclast system activation and the propionic acid generated directly by P. acnes. The altered loading situation, together with the degenerating discs, can activate osteoclastic progress resulting in bone degenerative changes in the endplate. As a major metabolite of $\mathrm{P}$. acnes, propionic acid could also be responsible for the osteolysis (13).

Albert et all. (14) have proposed antibiotic treatment in patients with chronic low back pain and Modic type 1 changes (bone edema). They recruited patients whose only known illness was chronic LBP of greater than 6 months duration occurring after a previous disc herniation and who also had bone edema demonstrated as Modic type 1 changes in the vertebrae adjacent to the previous herniation. These patients were randomized to either 100 days of antibiotic treatment with amoxicillin-clavulanate or placebo and were blindly evaluated at baseline, end of treatment and at 1-year follow-up. The antibiotic group improved highly statistically significantly on all outcome measures and improvement continued from 100 days follow-up until 1-year follow-up. The antibiotic protocol in this study was significantly more effective for this group of patients than placebo in all the outcomes.

We think that 100 days with antibiotics, could have a lot of secondary effects, and we know after 16 years of experience with ozone therapy that this therapeutic has anti-inflammatory and analgesic properties, a powerful anti-microbial effect. It's an immuno-modulator and oxidative stress modulator $(15,16,17)$.

With all of this in mind we propose ozone therapy to treat Modic Changes in degenerative disc disease.

\section{Objectives}

Evaluate the administration of intradiscal injections of ozone therapy to "kill" the P. acnes and treat the inflammation around the endplate. We propose 3 intradiscal (because we always did two) and patients with Modic had more resistance to the treatment and minor response.

\section{Materials and Methods}

\section{Inclusion criteria}

Men or non-pregnant women with LBP (6 months duration) and Modic changes Type 1 confirmed by NMR of lumbar spine L3/L4 or L4/L5 or L5/S1. Patients who mark pain greater than or equal to 4 in the Visual Analogue Scale (VAS) and/ or deconditioning due to pain greater than $40 \%$ in the ODI scale (The Oswestry Disability Index). Age: between 18 and 75 years. Weight: greater than $45 \mathrm{Kg}$. Cardiac, liver, respiratory and renal functions must be compensated. Both conservative and surgically treated patients were included. All the patients fulfilled these criteria were posted a letter describing the project as well as a questionnaire containing pain drawings.

\section{Exclusion criteria}

Current pregnancy or lactation, any kidney or liver disease, or active neoplasm. Viral, bacterial or fungal active systemic infection at the time of admission.

\section{Admission}

Between February 2016 and august 2018 we recruited 170 patients. 50 had Modic Changes type1 and get into this work. 21 were women and 29 men. Ages were between 24 and 73 years old. They manifested 4 or more pain on VAS and $40 \%$ (or more) in ODI. Informed consent was taken from all the paticipants and they were examined physically and clinically at admission. 
They registered their pain on the VAS before starting the treatment, and they completed the ODI scale in its version validated for Argentina. The patients performed these same questionnaires at the end of the treatment.

\section{Registry}

The records of the VAS and ODI scales; the photos of the MRI and all the clinical data were stored in a database, by a medical professional blind to the intervention.

\section{Treatment}

We used an Ozomed® portable (Kastber Praxisbedarf GMBH-Medizintechnik). All the patients received 10 sessions of $\mathrm{O} 2-\mathrm{O} 3$ ambulatory twice a week. These applications consisted of injections in the paravertebral muscles corresponding to the affected metamers. We injected $10 \mathrm{ml}$ at a concentration of $10 \mu \mathrm{g} / \mathrm{ml}$ in each metamer. Also, we did three intradiscal applications in an operating room at "La Sagrada Familia" clinic, under fluoroscopic control and with neuroleptoanesthesia (propofol, fentanyl), in which $60 \mathrm{ml}$ of the $\mathrm{O} 2 \mathrm{O} 3$ mixture was applied at a concentration of $43 \mu \mathrm{g} / \mathrm{ml}$.

All patients underwent a control MRI, six months after the end of treatment.

\section{Results}

The treatment was considered successful when the difference between the VAS at the beginning of the treatment and the VAS at the end of the treatment was greater than or equal to 4 points. 43 patients obtained 4 or more points of difference at the end of the treatment and that's means $86 \%$ success (graphic 1). The initial VAS average was 7.76 and the final VAS average was 1.9. The devest was 1.61 and 1.51 respectively. We don't have enough patients at all and our data has dispersion. However, the difference between the initial VAS average and the final VAS average is significant (graphic 2). We didn't get such good results with ODI scale. Only $50 \%$ get better in ODI scale of which $15 \%$ went from intense to moderate, $15 \%$ from intense to minimum, $10 \%$ from incapacity to minimum, $10 \%$ from moderate to minimum. $50 \%$ didn't change ODI scale (graphic 3). The MRI images didn't show significant difference between the beginning and the end of the treatment. However, it could be notice certain decrease in edema around the endplate (with Modic changes) and better definition at the edges of this endplates on control MRI after six months of the treatment. 
Graphic 1

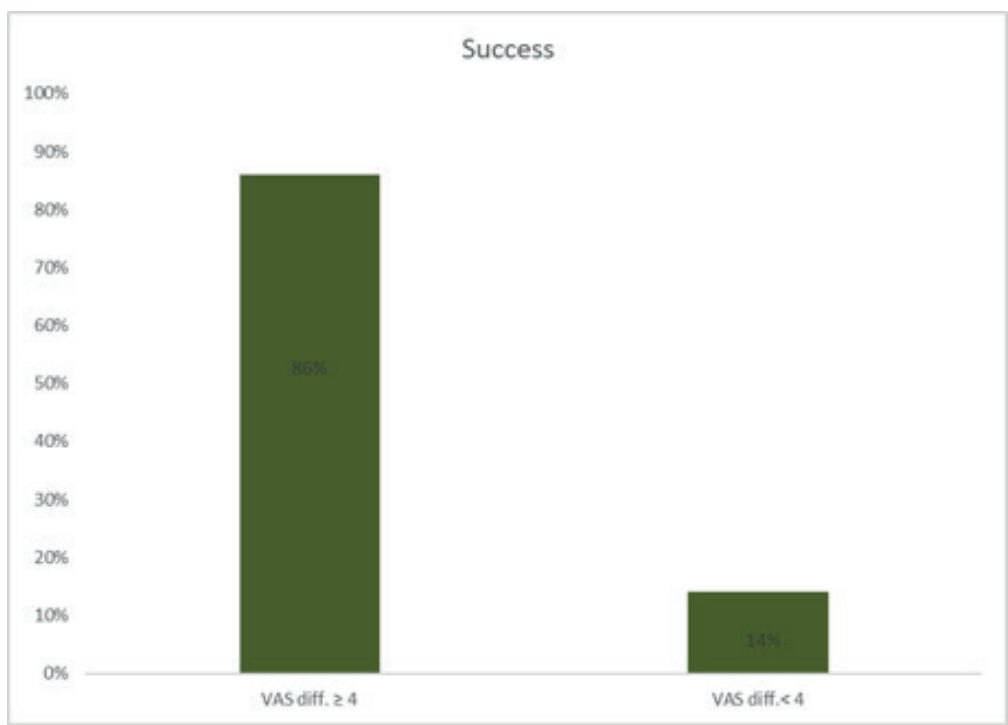

Graphic 2

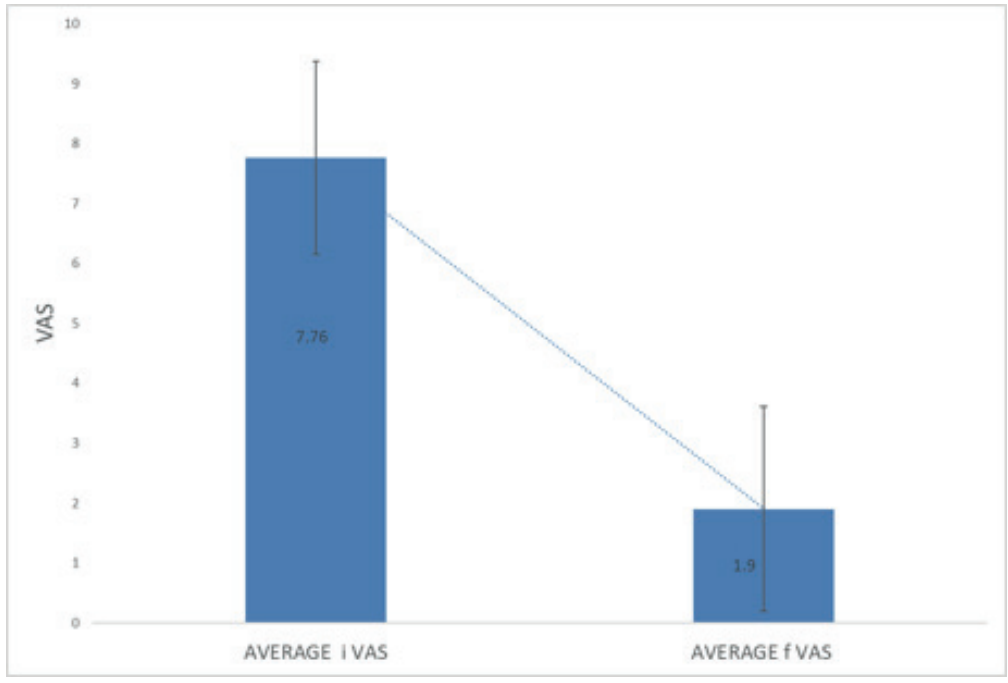

Graphic 3

$60 \%$

Response to treatment on ODI scale

$50 \%$

$40 \%$

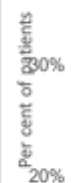

$15 \%$

$10 \%$

$0 \%$

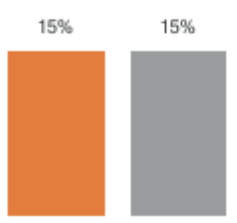

$10 \%$

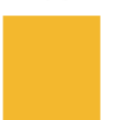

$10 \%$

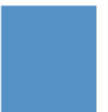

- intense to moderate

minterse to minimun

"incapacity to minimun

- moderate to minimun

- no change 


\section{Discussion}

Low back pain has been associated with degeneration of the intervertebral disc and by inference linked to aging, excessive manual labor and more recently to genetic factors. It is estimated that as much as $84 \%$ of the population suffers from low back pain at some point in their lifetime (18), while $10 \%$ are chronically disabled. The socio-economic cost of the condition is enormous, estimated to be $\$ 85$ billion in 2008; in the UK, in terms of lost productivity, disability benefits total more than $£ 12$ billion. As such, lower back pain is one of the most prevalent musculoskeletal conditions affecting Western society and a huge drain on medical resources worldwide. A widely recognized contributor to back pain is degeneration of the intervertebral disc. (18-20),

The intervertebral disc (IVD) is composed of the nucleus pulposus (NP) centrally, the annulus fibrosus (AF) peripherally, and the cartelaginous endplates cranially and caudally at the junction to the vertebral bodies. Within the NP, an abundance of proteoglycans allows for absorption of water. This property of the NP is essential for the IVD's handling of axial loads. In the healthy disc, the most common type of collagen within the NP is type II collagen. The AF surrounds the NP and consists primarily of type I collagen.

Although many conditions lead to chronic low back pain, degenerative disc disease (DDD) is among the most common diagnoses. Because correlations among clinical symptoms, radiological signs of disc degeneration, and treatment outcomes are not satisfactory, the selection of treatment options is difficult and the options employed often yield less than satisfactory results. Clearly, a more detailed understanding of the causes and development of DDD is needed so patients can be stratified for tailored or personalized treatments where possible (21).

Disc degeneration refers to the structural and functional failure of the disc as a result of aberrant, pathological cellular and extra cellular matrix changes. Disc degeneration may be caused by genetic predisposition, injury, aging, and environmental factors such as smoking, or any combination thereof. It is not exclusive to the older population, can be present in a younger person due to injury or faulty. Chronic activation of NF-kB has been linked to tissue aging and many age-related degenerative diseases, including musculoskeletal disorders such as muscular dystrophy, osteoarthritis, and osteoporosis. NF-kB is also implicated age-associated intervertebral disc degeneration.

In disc tissue, NF-KB activity was shown to correlate with accumulated oxidative stress and increase with age and degeneration. Several studies focus on the role of NF-KB in mediating degenerative and inflammatory disc disease. Increased NF-KB activity is found in degenerative discs and these discs have higher levels of pro-inflammatory cytokines that are considered typical NF-kB target genes, TNF-a, IL-1 $\beta$, IL-6 and IL-8. Furthermore, Mitogen-Activated Protein Kinases (MAPKs) are a family of signal transduction pathways, allowing the cells to respond to multiple extracellular inputs, such as hormones, growth factors, inflammatory cytokines, and environmental stresses such as ionizing radiation or osmotic stress. Multiple components of the catabolic machinery are regulated by MAPK family members. Many of these catabolic genes are also regulated by NF-KB signaling. In disc tissue, the major pro-inflammatory cytokines (IL-1 $\beta$ and TNF-a) activate ERK and/or p38 and consequently catabolic molecules. Inhibition of MAPK activation prevent these processes, indicating that MAPK regulation could mitigate disc degeneration (22)

The harsh microenvironment of degenerative discs is characterized by low nutrition, high levels of cytokines and oxidative stress. Oxidative stress is a major contributor to cellular senescence. The levels of ROS in discs increased with 
IDD advancing. The levels of pro-inflammatory cytokines in degenerative discs increased with IDD progressing too. These cytokines (TNF-a, IL-1a, IL-1b, IL$6, \mathrm{IL}-17$, and various chemokines) are widely accepted mediators of IDD, which enhance the extra cellular matrix catabolism and inflammation in discs, leading to the structural and functional deterioration of discs. Recently, the effects of cytokines on disc cell senescence have been investigated. TNF-a and IL-1b, are powerful pro-senescence factors in degenerative discs (23).

On the other hand, there is an association between degenerative disc disease and Modic changes.

In a study with 412 persons aged 40 from the general population a strong association with LBP within the past year, particularly for MC type 1 . The prevalence of pain within the last year in people with $\mathrm{MC}$ was $88 \%$ and in people with no pain $12 \%$ (24) In another study with patients with sciatica (92\% from a lumbar disc herniation) all patients had an MRI in the acute stage and at follow-up 14 months later. At follow-up, $60 \%$ of the patients with MC suffered from LBP, whereas in the group without MC only $20 \%$ had LBP. The prevalence of MC increased in the 14 months from $25 \%$ to $49 \%$. Lumbar pain was more frequent in people with MC type 1 compared to those with type 2, although not statistically significant. (25). The reason why MC type 1 are more painful could be that they reflect an earlier and more active stage of inflammation (26).

Signal changes in the vertebral endplate and vertebral bone marrow are on the other hand closely linked to low back pain. Disc herniation and severe disc degeneration disc are strong risk factor for developing MC (especially type 1). The strong association between MC, severely degenerated discs and previous disc herniation, gives reasons to propose the hypothesis that $\mathrm{MC}$ may be caused by mechanical stress. In case of a herniation and severe degeneration the loss of nuclear material may increase the shear forces on the endplates and micro fractures may occur (26). And, as we say before, repeated trauma to intervertebral disks causes release of inflammatory mediators within the nucleus pulposus that then diffuse through the vertebral endplates into the adjacent marrow instigating the inflammatory process (8).

Another patho-genetic reason for the occurrence of $\mathrm{MC}$ might be an infection involving low virulent anaerobic bacteria following a breach of the outer annulus fibrous, and an inflammation with a presence of macrophages occurs. This special environment, it might be possible for the anaerobic bacteria to enter the disc through the breach, causing a low virulent and slowly developing infection in the disc. Due to the fact that the disc is an avascular structure, it is an ideal environment for the growth of anaerobic bacteria. The infection may therefore result in local tissue inflammation with edema, and due to production of cytokines known to affect the bone; this is leading to MC type 1 in the vertebral endplates and subchondral bone marrow (26). The identification of Propionibacterium acnes biofilms in intervertebral disc tissue obtained from patients who have undergone microdiscectomy, combined with previous studies demonstrating the success of antibiotic treatment for patients with CLBP (Albert et al., 2013), has led to the suggestion that $\mathrm{P}$. acnes infection of the intervertebral disc may be another important causative factor for the development of DDD (21).

Ozone (O3) gas, discovered in the mid-nineteenth century, is a molecule consisting of three atoms of oxygen. It is one of the most reactive gases and the third most potent oxidant after fluorine and persulfate. Ozone therapy has been used and studied for more than a century for its use as a disinfectant (against the harmful effects of bacteria, viruses, fungi, yeast and protozoa) and for the treatment of diseases (by stimulating the oxygen metabolism and activating the immune system). The first report on ozone therapy and classical applications 
of ozone treatment was published by Wolff in 1974. Ozone therapy effects has proven to be consistent, safe and with minimal and preventable side effects when administrated within the appropriate dosage and manner (27). There is extensive description of what ozone is and its biological effects elicited by its messengers, such as reactive oxygen species (ROS) and lipid oxidative products (LOPs), and its therapeutic effects. Ozone immediately reacts as soon as it is dissolved in biological water (physiological saline, plasma, lymph, urine, interstitial fluid). Ozone reacts with both present antioxidants and polyunsaturated fatty acids (PUFAs) (28). O3 can cause this mild oxidative stress because of its ability to dissolve in the aqueous component of plasma. By reacting with polyunsaturated fatty acids (PUFA) and water, $\mathrm{O} 3$ creates hydrogen peroxide $(\mathrm{H} 2 \mathrm{O} 2)$, a reactive oxygen species (ROS). Simultaneously, O3 forms a mixture of lipid ozonation products (LOP). The LOPs created after $\mathrm{O} 3$ exposure include lipoperoxyl radicals, hydroperoxides, malonyldialdeyde, isoprostanes, the ozonide and alkenals, and 4-hydroxynonenal (4-HNE). Moderate oxidative stress caused by $\mathrm{O} 3$ increases activation of the transcriptional factor mediating nuclear factor-erythroid 2-related factor 2 (Nrf2). Nrf2's domain is responsible for activating the transcription of antioxidant response elements (ARE). Upon induction of ARE transcription, an assortment of antioxidant enzymes gains increased concentration levels in response to the transient oxidative stress of $\mathrm{O} 3$. The antioxidants created include, but are not limited to, superoxide dismutase (SOD), glutathione peroxidase (GPx), glutathione S-transferase (GST), catalase (CAT), heme oxygenase-1 (HO-1), NADPHquinone-oxidoreductase (NQO-1), heat shock proteins (HSP), and phase II enzymes of drug metabolism. Many of these enzymes act as free radical scavengers clinically relevant to a wide variety of diseases (17).

On the other hand, ozone has antimicrobial properties. When bacteria are exposed to $\mathrm{O} 3$ in vitro, the phospholipids, and lipoproteins that are within the bacterial cell envelope are oxidized. As this occurs, the stability of the bacterial cell envelope is attenuated. Moreover, evidence has demonstrated $\mathrm{O} 3$ to interact with fungal cell walls like bacteria. This disrupts the integrity of the cytosolic membrane and infiltrates the microorganisms to oxidize glycoproteins, glycolipids, and block enzymatic function. The combination of these reactions causes inhibition of fungi growth and mortality of bacteria and fungi (17). Ozone has been used for treatment of open wounds, Herpes Zoster and Herpes Simplex, because of its anti-microorganism effectiveness. It's efficient in killing many kinds of microorganisms, such as S. aureus, Streptococci spp, Escherichia coli, Enterococcus faecalis, and P. aeruginosa. Studies also have shown that ozone therapy can disinfect against $\mathrm{S}$. aureus and methicillin resistant Staphylococcus aureus strain in vitro. In vivo studies have suggested that ozone therapy is safe and exhibits antibacterial effects for the treatment of peritonitis. Ozone has shown its efficacy on healing methicillin resistant Staphylococcus aureus skin infections when combined with other drugs (29).

\section{Conclusion}

In our work patients who were treated with ozone therapy according to our treatment proposal showed a substantial improvement on VAS comparing the beginning and the end of the treatment. They show a significant difference in pain, and general condition. However, these results were not reflected on ODI scale. It's important to say that changes were not so strong on the MRI but we could notice certain improvements. For example, we could see a decrease in edema around the endplate with Modic changes and better definition at the edges of the endplates after six months of the end of the treatment.

Respect to the results in the ODI scale, this index, as its name implies, evaluates 
disability or deconditioning due to pain. This scale is directly related to the patient's previous sequelae, which are what determine his disability or deconditioning. Pain responds quickly to treatments with ozone therapy, however recovery from deconditioning is subsequent to the remission of pain that we measure with the VAS scale. Moreover, many times this disability does not recover, but patients don't feel pain. For this reason, we recommend our patients to accompany the ozone therapy treatment with rehabilitation in order to correct these deconditioning. In conclusion, we think that ozone therapy is the best treatment for degenerative disc disease with Modic changes type 1 because avoids the advance of degenerative disc disease and the inflammatory processes, because decreases the oxidative stress which is one of the triggers of disc degeneration. It decreases the activation of the NFK $\beta$ and MAPKs, so mitigates the inflammatory mediators, senescence and apoptosis. And we think that intradiscal ozone kills P. acnes biofilm. All together leads to the clinical improvement of the patients, MCs and of course degenerative disc disease.

\section{Bibliography}

1. Yue-Hui Z, Chang-Qing Z, Lei-Sheng J, Xiao-Dong C, Li-Yang D. Modic changes: a systematic review of the literature. Eur Spine J. 2008; 17:1289-1299.

2. Modic MT, Steinberg PM, Ross JS, Masaryk TJ, Carter JR. Degenerative disk disease: assessment of changes in vertebral body marrow with MR imaging. Radiology. 1988; 166:193-199.

3. Michael TM, Jeffrey SR. Lumbar Degenerative Disk Disease. Radiology. 2007 Oct;225(1):43-61.

4. Albert HB, Lambert P, Rollason J, Solgaard J, Worthington T, Bach Mogens, Schack H, Vernallis A, Busch F, Manniche C, Elliott T. Does nuclear tissue infected with bacteria following disc herniations lead to Modic changes in the adjacent vertebrae? Eur Spine J. 2013;22:690-696.

5. Rollason J, McDowell A, Albert HB, Barnard E, Worthington T, Hilton AC, Vernallis A, Patrick S, Elliott T, Lambert P. Genotypic and Antimicrobial Characterisation of Propionibacterium acnes Isolates from Surgically Excised Lumbar Disc Herniations. Biomed Res Int. 2013;2013:1-7.

6. Stirling A, Worthington T, Rafiq M, Lambert PA, Elliott TSJ. Association between sciatica and Propionibacterium acnes. The Lancet. 2001 Jun 23;357:2023-2025.

7. Burke JG, Watson RW, McCormack D, Dowling FE, Walsh MG, Fitzpatrick JM. Intervertebral discs which cause low back pain secrete high levels of proinflammatory mediators. J Bone Joint Surg Br. 2002; 84:196-201.

8. Crock HV. Internal disc disruption: a challenge to disc prolapse fifty years on. Spine. 1986;11(6):650-653.

9. Albert HB, Sorensen JS, Christensen BS, Manniche C. Antibiotic treatment in patients with chronic low back pain and vertebral bone edema (Modic type 1 changes): a double-blind randomized clinical controlled trial of efficacy. Eur Spine J. 2013;22:697-707.

10. Crockett MT, Sean B, van Baarsel KS, Kavanagh C. Modic Type 1 Vertebral Endplate Changes: Injury, Inflammation, or Infection? AJR. 2017;209(1):167-170. 11. Uquhart DM, Zheng Y, Cheng AC, Rosenfeld JV, Chan P, Liew S, Monira S, Cicuttini FM. Could low grade bacterial infection contribute to low back pain? A systematic review. BMC Medl. 2015 Jan 22;13:13.

12. Urquhart DM, Zheng Y, Cheng AC. Can bacterial infection by low virulent organisms be a plausible cause for symptomatic disc degeneration? A systematic review. Spine. 2015;40(10):E587-E592.

13. Shan Z, Zhang X, Li S, Yu T, Liu J, Zhao F. Propionibacterium acnes Incubation in the Discs Can Result in Time-Dependent Modic Changes. A Long-Term Rabbit 
Model. Spine. 2017 Nov 1;42(21):1595-1603.

14. Albert HB, Sorensen JS, Schiott B, Manniche C. Antibiotic treatment in patients with chronic low back pain and vertebral bone edema (Modic type 1 changes): a double-blind randomized clinical controlled trial of efficacy. Eur Spine J. 2013;22:697-707.

15. Re L, Mawsouf MN, Menendez S, Leon OS, Sanchez GM, Hernandez F. Ozone Therapy: Clinical and Basic Evidence of Its Therapeutic Potential. Arch Med Res. 2008;39(17):26.

16. Bocci V. How a calculated oxidative stress can yield multiple therapeutic effects. Free Radic Res. 2012 Sep.46(9):1068-1075.

17. Smith NL, Wilson AL, Gandhi J, Vatsia S, Khan SA. Ozone therapy: an overview of pharmacodynamics, current research, and clinical utility. Med Gas Res. 2017;7(3):212-219.

18. Risbud MV, Shapiro IM. Role of Cytokines in Intervertebral Disc Degeneration: Pain and Disc-content. Nat Rev Rheumatol. 2014 Jan;10(1):44-56.

19. Martin BI, Deyo RA, Mirza SK et al. Expenditures and health status among adults with back and neck problems. JAMA. 2008;299(6):656-664.

20. Stewart WF, Ricci JA, Chee E, Morganstein D, Lipton R. Lost productive time and cost due to common pain conditions in the US workforce. JAMA. 2003; 290:2443-2454.

21. Slaby O, McDowell A, Brüggemann H, Raz A, Demir-Deviren S, Freemont $T$, Lambert P, Capoor MN. Is IL-1b Further Evidence for the Role of Propionibacterium acnes in Degenerative Disc Disease? Lessons From the Study of the Inflammatory Skin Condition Acne Vulgaris. Front Cell Infect Microbiol. 2018 Aug;8:272.

22. Vo NV, Hartman RA, Patil PR, Risbud MV, Kletsas D, latridis JC, Hoyland JA, Le Maitre CL, Sowa GA, Kang JD. Molecular Mechanisms of Biological Aging in Intervertebral Discs. J Orthop Res. 2016 Aug;34(8):1289-1306.

23. Feng C, Liu H, Yang M, Zhang Y, Huang B, Zhou Y. Disc cell senescence in intervertebral disc degeneration: Causes and molecular Pathways. Cell Cycle. 2016;15(13): 1674-1684.

24. Kjaer P, Leboeuf-Yde C, Korsholm L, Sorensen JS, Bendix T. A diagnostic imaging study of 40 year-old men and women. Magnetic resonance imaging and low back pain in adults. Spine. 2005; 30:1173-1180.

25. Albert HB, Manniche C. Modic changes following lumbar disc herniation. Eur Spine J. 2007 Mar 3;16(7):977-982.

26. Albert HB, Kjaer P, Jensen TS, Sorensen JS, Bendix T, Manniche C. Modic changes, possible causes and relation to low back pain. Med Hypotheses. 2008;70:361-368.

27. Mehraban F, Seyedarabi A, Seraj Z, Ahmadian S, Poursasan N, Rayati S, Moosavi-Movahedi AA. Molecular insights into the effect of ozone on human hemoglobin in autohemotherapy: Highlighting the importance of the presence of blood antioxidants during ozonation. Int J Biol Macromol. 2018 Nov;119:12761285.

28. Bocci V, Borrelli E, Zanardi I, Travagli V. The usefulness of ozone treatment in spinal pain. Drug Des Dev Ther. 2015;9:2677-2685.

29. Song M, Zeng Q, Xiang Y, Gao L, Huang J, Huang J, Wu K, Lu J. The antibacterial effect of topical ozone on the treatment of MRSA skin infection. Mol Med Rep. 2018;17:2449-2455. 